\title{
Mixing of CNO-cycled matter in massive stars ${ }^{\star}, \star \star$
}

\author{
N. Przybilla ${ }^{1}$, M. Firnstein ${ }^{1}$, M. F. Nieva ${ }^{2}$, G. Meynet ${ }^{3}$, and A. Maeder ${ }^{3}$ \\ 1 Dr. Karl Remeis-Observatory \& ECAP, Astronomical Institute, Friedrich-Alexander University Erlangen-Nuremberg, \\ Sternwartstr. 7, 96049 Bamberg, Germany \\ e-mail: przybilla@sternwarte.uni-erlangen.de \\ 2 Max-Planck-Institut für Astrophysik, Karl-Schwarzschild-Str. 1, 85741 Garching, Germany \\ ${ }^{3}$ Geneva Observatory, University of Geneva, Maillettes 51, 1290 Sauverny, Switzerland
}

Received 29 January 2010 / Accepted 17 April 2010

\section{ABSTRACT}

\begin{abstract}
Aims. We test predictions of evolution models on mixing of CNO-cycled products in massive stars from a fundamental perspective. Relative changes within the theoretical C:N:O abundance ratios and the buildup of helium are compared with observational results. Methods. A sample of well-studied Galactic massive stars is presented. High-quality optical spectra are carefully analysed using improved NLTE line-formation and comprehensive analysis strategies. The results are put in the context of the existing literature data. Results. A tight trend in the observed N/C vs. N/O ratios and the buildup of helium is found from the self-consistent analysis of main-sequence to supergiant stars for the first time. The catalytic nature of the CNO-cycles is confirmed quantitatively, though further investigations are required to derive a fully consistent picture. Our observational results support the case of strong mixing, as predicted e.g. by evolution models that consider magnetic fields or by models that have gone through the first dredge-up in the case of many supergiants.
\end{abstract}

Key words. stars: abundances - stars: atmospheres - stars: early-type - stars: evolution - stars: massive - supergiants

\section{Introduction}

Energy production in massive stars is governed by the CNOcycles throughout most of their lifetimes. The general correctness of our understanding of the $\mathrm{CNO}$ cycles and of the relevant nuclear data (e.g. Maeder 1983) is confirmed impressively by observation: when massive stars enter the Wolf-Rayet phase as WN subtypes, equilibrium CNO-processed material becomes exposed on their surface (see e.g. Crowther 2007).

However, traces of mixing of $\mathrm{CNO}$-cycled products from the stellar core to the stellar surface can already be found much earlier in the lives of massive stars. Observational indications of superficial abundance anomalies for carbon, nitrogen, and oxygen (and the burning product helium) in OB-type stars even on the main sequence (MS) and, more prominently, in the blue supergiants was found early in classification spectrograms (e.g. Walborn 1976). Subsequent analyses provided evidence for a characteristic enrichment of nitrogen - which is the easiest to be detected - and helium in many early-type stars, both near the MS and in blue supergiants (e.g. Schönberner et al. 1988; Gies \& Lambert 1992; Herrero et al. 1992; Kilian 1992; Venn 1995; Lyubimkov 1996; McErlean et al. 1999).

A theoretical understanding of early mixing of CNO-processed material to the stellar surface could not be achieved within the framework of evolution calculations for non-rotating stars with mass-loss, which were state-of-the-art at that time (e.g. Chiosi \& Maeder 1986). The pollution of the surface layers with CNO-cycled material only occurs when the star reaches the red supergiant phase in such models, via convective dredge-up.

$\star$ Based on observations obtained at the European Southern Observatory, proposals $62 . \mathrm{H}-0176 \&$ 074.B-0455(A).

$\star \star$ Based on observations collected at the Centro Astronómico Hispano Alemán at Calar Alto, proposals H2001-2.2-011 \& H2005-2.2-016.
Considered a secondary effect for a long time, stellar rotation has come lately into focus. It turned out that rotationally-induced mixing through meridional circulation and turbulent diffusion in rotating stars provides the means to change all model outputs substantially and to bring theory and observation into much better agreement (Maeder \& Meynet 2000; Heger \& Langer 2000). The latest step taken in the modelling was to consider the effects from an interplay of rotation and magnetic fields, which - depending on the detailed input physics and approximations made - only provide minor modifications to the surface abundances (Heger et al. 2005) or substantial changes (Maeder \& Meynet 2005, henceforth abbreviated as MM05). These differences result mainly from the two groups using different sets of equations for computing the effects of magnetic fields; see in particular, the changes brought to the system of equations given by Spruit (2002) by MM05 in their Sect. 2.

The only means to verify the models is via systematic comparison with observations covering the relevant parts of the Hertzsprung-Russell diagram. Homogeneous analyses of a larger star sample from the main sequence to the supergiant stage have only recently become available. The results in particular for $\mathrm{N}$ abundances apparently challenge the concept of rotational mixing in massive stars (Hunter et al. 2009) and thus the presentday evolution models (see, however, Maeder et al. 2009).

Here, we address the topic of early mixing of CNO-cycled products in massive stars from a fundamental perspective. We compare the model predictions for the relative changes within the C:N:O abundance ratios and the buildup of helium (Sect. 2) with observations. For this we briefly review the status of the literature on $\mathrm{CNO}$ abundances and introduce a well-studied Galactic star sample for which high-accuracy analyses of highquality spectra were performed using improved NLTE modelling and comprehensive analysis techniques (Sect. 3). Conclusions from this comparison are drawn in Sect. 4. 

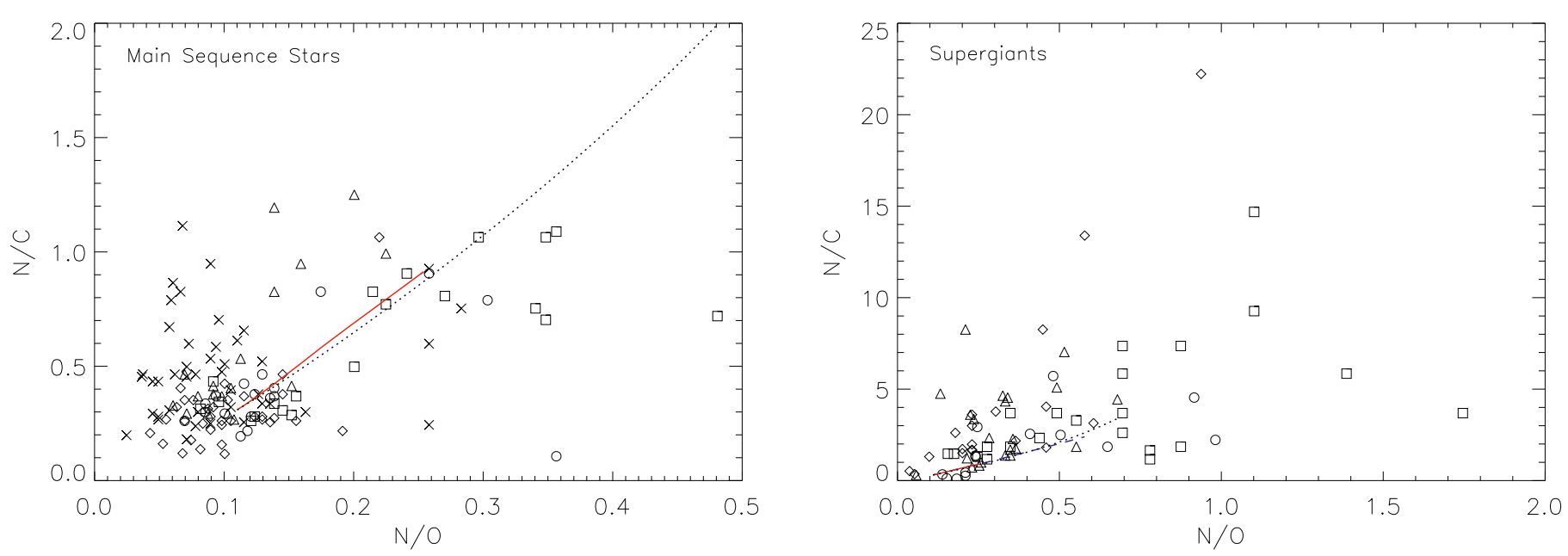

Fig. 1. Status of observational constraints on the (magneto-)hydrodynamic mixing of CNO-burning products in massive stars from previous NLTE analyses. Mass ratios N/C over N/O are displayed. Left panel: main-sequence stars. Circles: Kilian (1992); triangles: Gies \& Lambert (1992); diamonds: Cunha \& Lambert (1994), Daflon et al. (1999, 2001a,b); squares: Morel et al. (2008); crosses: Hunter et al. (2009). Right panel: BA supergiants. Triangles: Venn (1995), Venn \& Przybilla (2003); circles: Takeda (2000); squares: Crowther et al. (2006); diamonds: Searle et al. (2008). Error bars are omitted for clarity: uncertainties in the abundances of the individual elements are typically about a factor 2, such that the error bars can be larger than the plotting range. The lines represent predictions from evolution calculations, for a rotating $15 M_{\odot} s \operatorname{star}\left(v_{\mathrm{rot}}^{\text {in }}=300 \mathrm{~km} \mathrm{~s}^{-1}\right.$, Meynet \& Maeder 2003, MM03; until the end of the MS: solid red line, until the end of He burning: dashed blue line) and for a star of the same mass and $v_{\text {rot }}^{\text {ini }}$ that in addition takes the interaction of rotation and a magnetic dynamo into account (Maeder \& Meynet 2005, MM05; until the end of the MS: dotted line), respectively. The predicted trends are similar for the entire mass range under investigation, see Fig. 3.

\section{Theoretical considerations}

Diagnostic plots of an abundance ratio vs. another one, such as $\mathrm{N} / \mathrm{C}$ vs. N/O or helium vs. N/O discussed in this paper, depend on both the changes produced by nuclear reactions and the dilution effects produced by mixing. Let us estimate the slope $\frac{d(\mathrm{~N} / \mathrm{C})}{\mathrm{d}(\mathrm{N} / \mathrm{O})}$ produced by the nuclear effects at the beginning of CNO burning, with everything expressed in mass fractions. Apart from the very massive stars $\left(M>40 M_{\odot}\right)$, one may assume that, at the beginning of the burning, the ${ }^{14} \mathrm{~N}$ enhancement comes from the ${ }^{12} \mathrm{C}$ destruction via the $\mathrm{CN}$ cycle, and the oxygen content $\mathrm{O}$ remains about constant. Thus, one has $\mathrm{dC}=-\frac{6}{7} \mathrm{dN}$ since ${ }^{14} \mathrm{~N}$ globally results from the addition of two protons to ${ }^{12} \mathrm{C}$,

$\mathrm{d}(\mathrm{N} / \mathrm{O})=\mathrm{dN} / \mathrm{O}$

$\mathrm{d}(\mathrm{N} / \mathrm{C})=\frac{\mathrm{dN}}{\mathrm{C}}-\frac{\mathrm{N}}{\mathrm{C}^{2}} \frac{\mathrm{dC}}{\mathrm{dN}} \mathrm{dN}=\frac{\mathrm{dN}}{\mathrm{C}}\left[1+\frac{6}{7} \frac{\mathrm{N}}{\mathrm{C}}\right]$.

This gives the slope

$\frac{\mathrm{d}(\mathrm{N} / \mathrm{C})}{\mathrm{d}(\mathrm{N} / \mathrm{O})}=\frac{(\mathrm{N} / \mathrm{C})}{(\mathrm{N} / \mathrm{O})}\left[1+\frac{6}{7} \frac{\mathrm{N}}{\mathrm{C}}\right]$,

always in mass fractions. With initial ${ }^{1}$ ratios $\mathrm{N} / \mathrm{C}$ and N/O of 0.31 and 0.11 , respectively, we get

$\frac{\mathrm{d}(\mathrm{N} / \mathrm{C})}{\mathrm{d}(\mathrm{N} / \mathrm{O})}=3.77$.

This ratio is evidently greater than 1 , since as $\mathrm{N}$ starts growing, $\mathrm{C}$ decreases, while $\mathrm{O}$ does not vary much. The relation turns slightly upward as N/C is increasing owing to the term in brackets in Eq. (2). However, at some advanced stage in evolution, corresponding to WN stars not shown here, the curve will saturate and turn down slightly (Maeder 2009, p. 699), since the

\footnotetext{
1 The chemical composition of the evolution models is identical to those used in the OPAL opacity tables: the solar mixture of Grevesse \& Noels (1993).
}

$\mathrm{CN}$ cycle is then at equilibrium, while ${ }^{16} \mathrm{O}$ is still turned to ${ }^{14} \mathrm{~N}$. Dilution mixes a fraction $f$ of $\mathrm{N}+\Delta \mathrm{N}$ enriched and $\mathrm{C}$ depleted materials with a fraction $(1-f)$ of the original $\mathrm{N}$ and $\mathrm{C}$. Under the same assumptions as above, it is easy to show that, to the first order, the slope for the relative enrichments in the N/C vs. N/O plot behaves the same way as in Eq. (2) independently of $f$.

The value of $f$, however, determines the amplitudes of the departures from the cosmic ratios. Our models with rotational mixing (Meynet \& Maeder 2003, MM03; Ekström et al. 2008, E08) or with rotation and magnetic fields (MM05), as illustrated e.g. in Fig. 1, have an initial slope $\frac{\mathrm{d}(\mathrm{N} / \mathrm{C})}{\mathrm{d}(\mathrm{N} / \mathrm{O})} \approx 4$, which is in excellent agreement with Eq. (3). The amplitude $f$ of the mixing depends on the various model assumptions, in particular on the treatment of the shear mixing with or without horizontal turbulence. The models without horizontal turbulence (Meynet \& Maeder 2000, MM00) predict more mixing than models that account for it (MM03). Models that include both rotation and magnetic field predict a still larger mixing (MM05).

Let us now consider the behaviour of the helium surface content $Y_{\mathrm{s}}$ vs. N/O (as illustrated later). Strictly and only at the very beginning of the $\mathrm{CN}$ burning, and under the assumption of an initially constant oxygen, we get $\mathrm{d} Y_{\mathrm{s}}=\frac{2}{7} \mathrm{dN}$, since when 4 units of mass of helium are made, 14 units of mass of nitrogen are produced. The slope is

$\frac{\mathrm{d} Y_{\mathrm{s}}}{\mathrm{d}(\mathrm{N} / \mathrm{O})}=\frac{2}{7} \mathrm{O} \approx 0.286 \times 0.009=0.0026$

i.e., it is essentially flat initially. Later in the evolution, both $\mathrm{N}$ and $\mathrm{O}$ change simultaneously, and one has to rely on numerical models. The resulting slope in the models can vary; e.g., a steeper slope is inferred for the $25 M_{\odot}$ model of both MM03 and models by MM00 than for the $15 M_{\odot}$ models of MM03 and MM05. This depends on whether the matter that arrives at the surface comes from inner regions that are at both $\mathrm{CN}$ and $\mathrm{ON}$ equilibria, or only at $\mathrm{CN}$ equilibrium. There are, of course, a range of intermediate cases. 
N. Przybilla et al.: Mixing of CNO-cycled matter in massive stars

Table 1. Stellar parameters and elemental abundances of the sample stars.

\begin{tabular}{|c|c|c|c|c|c|c|c|c|c|}
\hline Star & $\begin{array}{l}T_{\text {eff }} \\
(\mathrm{K})\end{array}$ & $\begin{array}{l}\log g \\
\text { (cgs) }\end{array}$ & $\begin{array}{r}v \sin i \\
\left(\mathrm{~km} \mathrm{~s}^{-1}\right)\end{array}$ & $\begin{array}{r}Y_{\mathrm{S}} \\
\text { (by mass) }\end{array}$ & $\varepsilon(\mathrm{C})^{1}$ & $\varepsilon(\mathrm{N})$ & $\varepsilon(\mathrm{O})$ & $\varepsilon\left(\sum \mathrm{CNO}\right)$ & $M / M_{\odot}$ \\
\hline \multicolumn{10}{|c|}{ B-type main-sequence stars: } \\
\hline HD 36591 & $27000 \pm 300$ & $4.12 \pm 0.05$ & $12 \pm 1$ & $0.28 \pm 0.03$ & $8.33 \pm 0.08$ & $7.75 \pm 0.09$ & $8.75 \pm 0.11$ & $8.92 \pm 0.07$ & 13 \\
\hline HD 61068 & $26300 \pm 300$ & \pm 0.05 & $14 \pm$ & $28 \pm 0.03$ & $7 \pm$ & $00 \pm 0.12$ & $5 \pm 0.09$ & & 12 \\
\hline HD 63922 & $31200 \pm 300$ & $3.95 \pm 0.05$ & $29 \pm 4$ & $0.25 \pm 0.03$ & $34 \pm 0.08$ & $7.77 \pm 0.08$ & $8.79 \pm 0.10$ & 0.07 & 19 \\
\hline HD 74575 & $22900 \pm 300$ & $3.60 \pm 0.05$ & $11 \pm 2$ & $0.28 \pm 0.03$ & $8.37 \pm 0.10$ & $7.92 \pm 0.10$ & $8.80 \pm 0.08$ & $8.98 \pm 0.06$ & 12 \\
\hline HD 122980 & $20800 \pm 300$ & $4.22 \pm 0.05$ & $18 \pm 1$ & $0.28 \pm 0.03$ & $8.32 \pm 0.09$ & $7.76 \pm 0.08$ & $8.72 \pm 0.05$ & $8.90 \pm 0.04$ & 8 \\
\hline HD 149438 & $32000 \pm 300$ & $4.30 \pm 0.05$ & $4 \pm 1$ & $0.28 \pm 0.03$ & $8.30 \pm 0.12$ & $8.16 \pm 0.12$ & $8.77 \pm 0.08$ & $8.97 \pm 0.06$ & 17 \\
\hline \multicolumn{10}{|c|}{ BA-type supergiants: } \\
\hline HD 13476 & $8500 \pm 150$ & $1.40 \pm 0.10$ & $12 \pm 2$ & $0.36 \pm 0.08$ & $8.18 \pm 0.11$ & $8.58 \pm 0.04$ & $8.63 \pm 0.06$ & $8.98 \pm 0.03$ & 16 \\
\hline HD 14433 & $9150 \pm 150$ & $1.40 \pm 0.10$ & $17 \pm 3$ & $0.32 \pm 0.07$ & $8.23 \pm 0.04$ & $8.23 \pm 0.03$ & $8.67 \pm 0.05$ & & 19 \\
\hline HD 34085 & $12100 \pm 150$ & $1.75 \pm 0.10$ & $25 \pm 3$ & $0.32 \pm 0.04$ & $8.23 \pm 0.09$ & $8.47 \pm 0.06$ & $8.75 \pm 0.05$ & 0.03 & 23 \\
\hline HD 87737 & $9600 \pm 150$ & $2.00 \pm 0.10$ & $2 \pm 2$ & $0.37 \pm 0.03$ & $8.25 \pm 0.06$ & $8.52 \pm 0.08$ & $8.73 \pm 0.06$ & 0.04 & 10 \\
\hline HD 92207 & $9500 \pm 200$ & $1.20 \pm 0.10$ & $30 \pm 5$ & $0.35 \pm 0.06$ & $8.33 \pm 0.08$ & $8.25 \pm 0.04$ & $8.79 \pm 0.07$ & .04 & 27 \\
\hline HD 111613 & $9150 \pm 150$ & $1.45 \pm 0.10$ & $17 \pm 2$ & $0.35 \pm 0.05$ & $8.29 \pm 0.10$ & $6 \pm 0.04$ & $8.73 \pm 0.04$ & 03 & 17 \\
\hline HD 1 & $9300 \pm 250$ & $1.60 \pm 0.15$ & $15 \pm 6$ & $0.32 \pm 0.05$ & $8.29 \pm 0.09$ & $9 \pm 0.04$ & $3 \pm 0.02$ & 02 & 15 \\
\hline HD 1 & $9200 \pm 150$ & \pm 0.10 & 3 & $0.38 \pm 0.06$ & \pm & \pm 0 & \pm 0.04 & 9. & 11 \\
\hline HD 1 & $8700 \pm 150$ & $1.20 \pm 0.10$ & $10 \pm$ & $0.37 \pm 0.04$ & $8.09 \pm 0.07$ & $8.56 \pm 0.07$ & $8.69 \pm 0.04$ & 8.9 & 22 \\
\hline HD 202850 & $10800 \pm 200$ & $1.85 \pm 0.10$ & $14 \pm 5$ & $0.38 \pm 0.06$ & $8.16 \pm 0.04$ & $8.70 \pm 0.06$ & $8.76 \pm 0.05$ & 9.09 & 15 \\
\hline HD 207673 & $9250 \pm 100$ & $1.80 \pm 0.10$ & $1 \pm 2$ & $0.33 \pm 0.08$ & $8.17 \pm 0.09$ & $8.48 \pm 0.03$ & $8.72 \pm 0.04$ & $8.99 \pm 0.03$ & 12 \\
\hline HD 210221 & $8400 \pm 150$ & $1.40 \pm 0.10$ & $0 \pm 0$ & $0.34 \pm 0.03$ & $8.22 \pm 0.06$ & $8.52 \pm 0.06$ & $8.70 \pm 0.05$ & 9.00 & 15 \\
\hline HD 212593 & $11200 \pm 200$ & $2.10 \pm 0.10$ & 6 & $0.35 \pm 0.05$ & $8.30 \pm 0.08$ & $8.44 \pm 0.06$ & $8.74 \pm 0.04$ & 9.01 & 12 \\
\hline HD 213470 & $8400 \pm 150$ & $1.30 \pm 0.10$ & $13 \pm 1$ & $0.32 \pm 0.07$ & $8.17 \pm 0.08$ & $8.54 \pm 0.04$ & $8.65 \pm 0.03$ & $8.97 \pm 0.02$ & 18 \\
\hline
\end{tabular}

Notes. ${ }^{(1)} \varepsilon(X)=\log X / \mathrm{H}+12$.

\section{Observational constraints}

Numerous studies of $\mathrm{CNO}$ abundances in massive stars of the Milky Way are available from the literature, mostly for early Btype stars close to the MS and for B and A-type supergiants. We illustrate the results of several key publications in the N/O-N/C diagrams of Fig. 1. Some of the more recent studies (Crowther et al. 2006; Searle et al. 2008; Hunter et al. 2009) are based on NLTE model atmospheres, while the bulk of the data were obtained from NLTE line-formation computations on LTE model atmospheres - which is equivalent to the full NLTE approach in the cases under consideration (Nieva \& Przybilla 2007, NP07).

The MS stars show a concentration around the solar ratios of $\mathrm{N} / \mathrm{C} \approx 0.3$ and $\mathrm{N} / \mathrm{O} \approx 0.1$. However, overall a wide range of $\mathrm{N} / \mathrm{O}-\mathrm{N} / \mathrm{C}$ combinations has been realised, with the deviations from the predictions increasing in the supergiants. On the one hand, this leaves room for broad interpretation - even more so if only one of the elements (like N) is considered - including the statement that some observational data points pose a challenge for the evolution models. On the other hand, most of the data are nevertheless consistent with the predictions, as the abundance uncertainties are very large. Typically, the statistical $1 \sigma$-error in abundance per element is about a factor $\sim 2$, and systematic uncertainties are often largely underestimated (for a discussion of this see Nieva \& Przybilla 2010) or even unaccounted for. The error bars in Fig. 1 are larger than the entire plotting range in many cases. In consequence, no definite conclusions can be drawn on the quality of the stellar evolution models from these data.

In past years a number of high-accuracy studies of massive stars in the solar neighbourhood have been published by us. Carefully analysed data are available on 6 slowly-rotating early B-type stars near the MS (Nieva \& Przybilla 2006, 2007, 2008; Przybilla et al. 2008, PNB08) and on 14 BA-type supergiants (Przybilla et al. 2006; Firnstein 2006; Schiller \& Przybilla 2008).
In brief, high-resolution $(R=40-48000)$ and high-S/N spectra $(S / N>300)$ with wide wavelength coverage and thorough continuum normalisation (obtained with FOCES@Calar Alto Observatory and FEROS@ESO/La Silla) were analysed using a hybrid NLTE approach (Przybilla et al. 2006; NP07). State-ofthe-art atomic input data were used in the modelling. In contrast to all previous work, multiple hydrogen lines, the helium lines, multiple metal ionisation equilibria and the stellar energy distributions were reproduced simultaneously in an iterative approach to determine the stellar atmospheric parameters. Chemical abundances were derived from analysis of practically the entire observable spectrum per element. The rewards of such a comprehensive, but time-consuming procedure are unprecedentedly small statistical error margins and largely reduced systematics. The relevant results - effective temperature $T_{\text {eff }}$, surface gravity $\log g$, projected rotational velocity $v \sin i$, surface helium abundance $Y_{\mathrm{S}}$, CNO abundances and their total, and the zero-age MS mass (derived under the assumption that the objects have evolved directly from the MS) - are summarised in Table 1 and visualised in Figs. 2-6. We re-ran the analysis of the supergiant targets for the present work, taking advantage of improved model grids. Our new results for these supergiants agree with the earlier ones within the uncertainties but are more accurate.

The sample is displayed in the $\log T_{\text {eff }}-\log g$-plane and compared to evolutionary tracks for rotating $\operatorname{stars}^{2}$ in Fig. 2. The stars have initial masses between about 9 to $25 M_{\odot}$. Stars close to the MS are apparently slow rotators, while the atmospheric expansion has erased any indication of the initial rotational velocity in the supergiants. There is good qualitative agreement between the observations and predictions for the N/C ratios, finding low values close to the initial (solar) ratio near the MS

2 The metal content (by mass) of the models is $Z=0.020$, while a value of 0.014 seems more appropriate for the massive star population in the solar neighbourhood (PNB08). No fundamental changes to our conclusions are expected to arise from this difference. The tracks will shift a bit, in particular the zero-age MS towards higher gravities. 


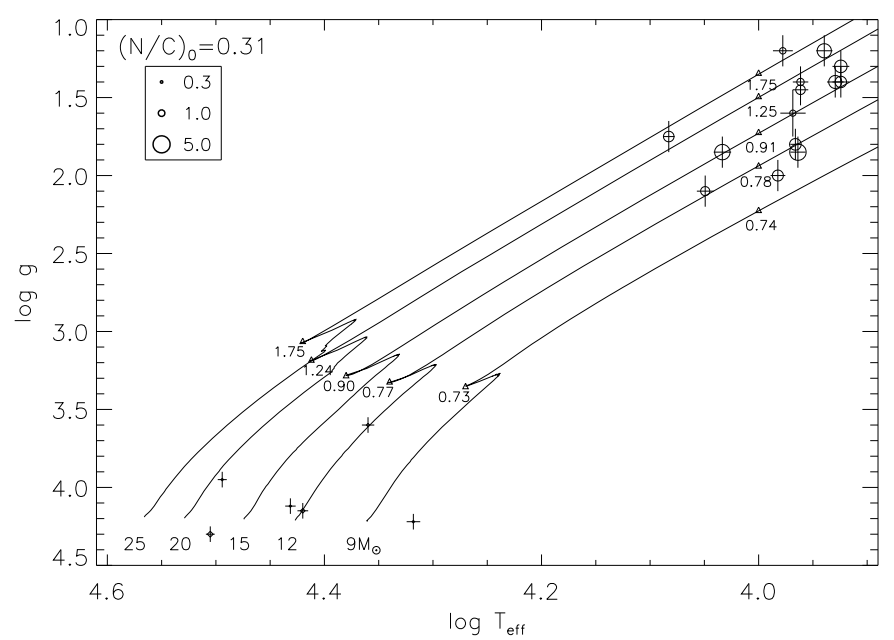

Fig. 2. Sample stars in the $\log T_{\text {eff }}-\log g$-plane. Diamonds denote MS stars and circles supergiants. The symbol size encodes the N/C ratio (by mass), with typical values exemplified in the legend. Evolutionary tracks for rotating stars of metallicity $Z=0.02$ (MM03) are shown. Predicted $\mathrm{N} / \mathrm{C}$ ratios are indicated along the tracks.

and enhanced values in the supergiants. However, the observed $\mathrm{N} / \mathrm{C}$ ratios reach much higher values than predicted by the models discussed in Fig. 2, provided these stars have recently left the MS and evolve now into red supergiants.

The behaviour of light element abundances of the MS sample stars is shown in Fig. 3. Theoretical evolutionary tracks are overplotted. It is striking to see that, regardless of the initial mass and of the initial rotation, the slope of the theoretical tracks is always the same and equal to the nuclear effect of the $\mathrm{CN}$ cycle as derived analytically in Sect. $2^{3}$. Thus the observed slope is a confirmation of the activity of the $\mathrm{CN}$ cycle. It does not depend on the stellar model but constitutes observational evidence of the nuclear path. As already indicated before, what is model dependent are the amplitudes of the departure from the cosmic ratios. Figure 3 shows that the observed points may be reproduced from models having differing velocities and masses, with and without magnetic field; however, by itself this diagram cannot help in disentangling the various possibilities.

The same evolutionary tracks are plotted in the N/C vs. $\log g$ diagram in Fig. 4. We see that, except for the stars HD 61068 and HD 149438 ( $\tau$ Sco, which is a genuine slow rotator with a rather strong magnetic field ${ }^{4}$ of probably fossil origin, Donati et al. 2006), the other points may be accounted for by models having low initial rotation. HD 61068 is marginally compatible with the $15 M_{\odot}$ model at $v_{\text {rot }}^{\text {ini }}=300 \mathrm{~km} \mathrm{~s}^{-1}$ computed with magnetic field, while $\tau$ Sco challenges present stellar models. It has a behaviour that may be explained by a homogeneous evolution, but this still has to be confirmed by further computations. This is a very interesting star that certainly deserves further inspections both from the observational and theoretical points of view.

The behaviour of light element abundances in the whole star sample is shown in Fig. 5. In contrast to the literature values

\footnotetext{
3 The theoretical slope varies between $\sim 3.0$ and 4.2 for typical initial CNO abundances (e.g. solar values according to Anders \& Grevesse 1989; Grevesse \& Sauval 1998; Asplund et al. 2009, or the cosmic abundance standard of PNB08), indicated by the grey area in Fig. 3. Improved agreement between observation and theory may be achieved when tailored abundances are used for the stellar evolution models, see also the previous footnote.

4 A magnetic field is present also in HD 74575 (Hubrig et al. 2009).
}

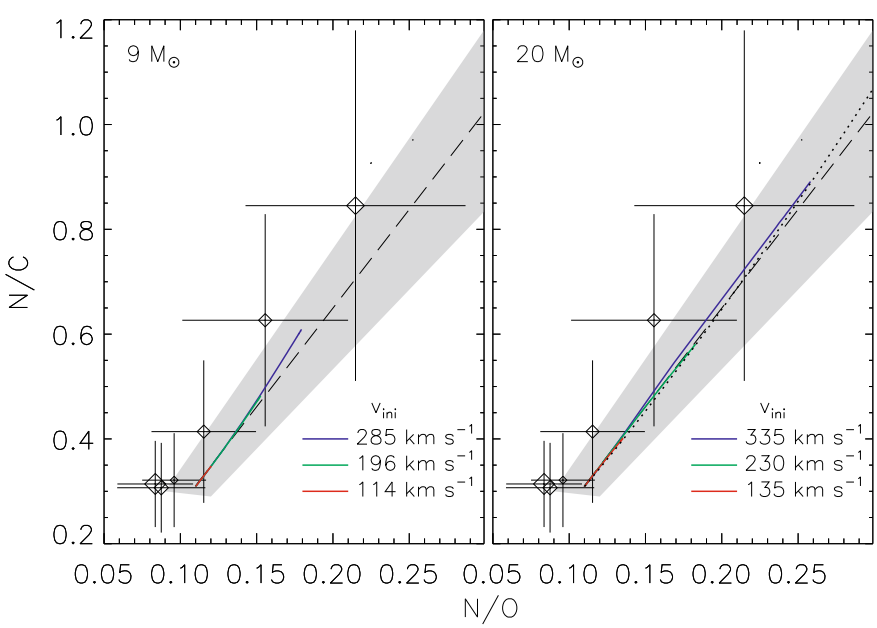

Fig. 3. Comparison of N/C vs. N/O abundance ratios (by mass) for our six MS sample stars (diamonds) with model predictions for $9 M_{\odot}$ (left) and $20 M_{\odot}$ stars (right panel) at different $v_{\text {rot }}^{\text {ini }}$ (see legend, equivalent to 30,50 and $70 \%$ of the star's breakup velocity, E08). The dotted line (right panel only) describes the magnetic $15 M_{\odot}$ model of MM05 with $v_{\mathrm{rot}}^{\mathrm{ini}}=300 \mathrm{~km} \mathrm{~s}^{-1}$. The symbol size encodes the stellar mass and error bars give $1 \sigma$-uncertainties. The long-dashed lines correspond to the theoretical slope 3.77 as deduced in Sect. 2. The grey area spans the full range of theoretical slopes using different references for solar abundances (see text, lower envelope defined by data of Asplund et al. 2009) and the cosmic abundance standard of PNB08 (upper envelope).

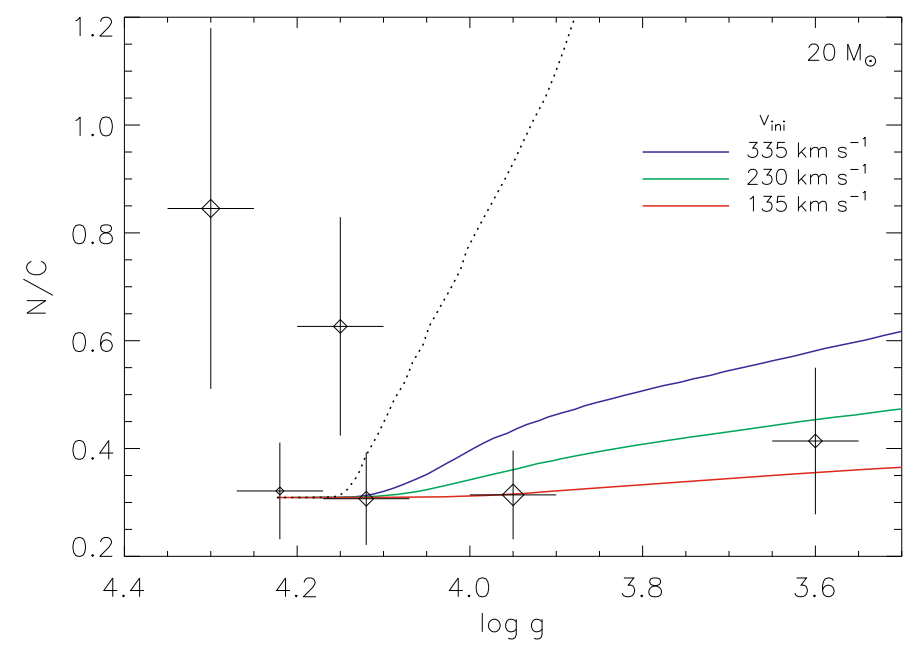

Fig. 4. N/C abundance ratios on the MS as function of $\log g$. The same tracks and observations as in Fig. 3 (right panel) are displayed.

(Fig. 1), a clear and tight trend is found, confirming the predicted locus of N/O-N/C abundance ratios. However, as already indicated above, the MM03 models for rotating stars with mass loss evolving towards the red supergiant stage (solid line in Fig. 5) predict mixing that is too low, i.e. too low $f$ (Sect. 2), in particular for most of the supergiants. A combination of five reasons may provide an explanation.

I) Higher than average rotation velocities in the progenitor stars of these supergiants on the MS may reconcile the situation for some objects.

II) Evolution models for rotating stars that also account for the interaction of rotation and a magnetic dynamo (MM05) predict enhanced mixing signatures of the amount required (dotted line). 


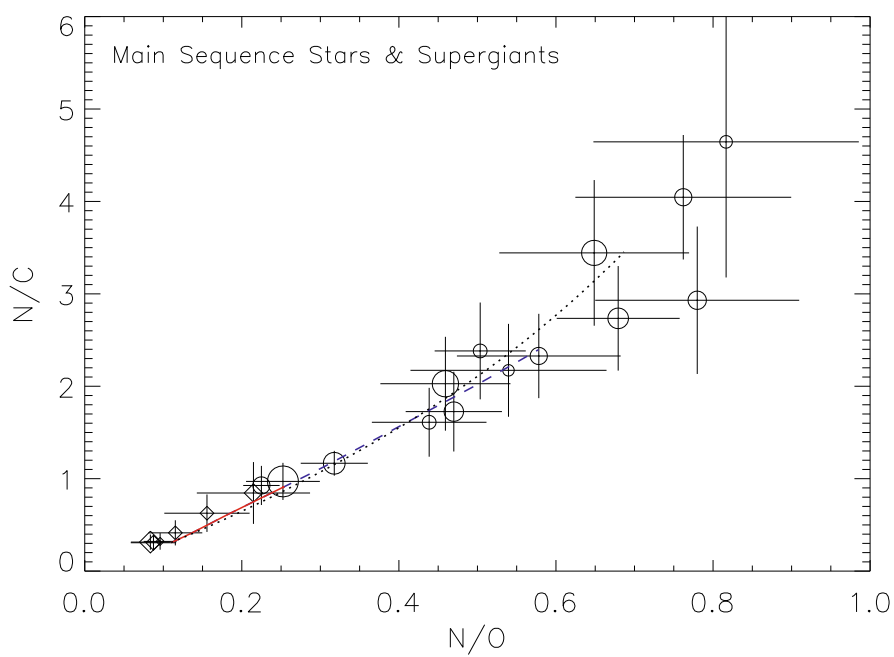

Fig. 5. N/C vs. N/O abundance ratios (by mass) for our sample stars. B-type MS stars are displayed as diamonds, BA-type supergiants as circles. The symbol size encodes the stellar mass and error bars give $1 \sigma$ uncertainties. The different lines describe model predictions for $15 M_{\odot}$ stars identical to those in Fig. 1.

III) Some stars may have evolved in a close binary, which can also lead to enhanced mixing associated with mass transfer.

IV) Some objects may have been siblings to $\tau$ Sco on the MS, climbing up the N/O-N/C relation even further in their further evolution.

v) Supergiants may already have evolved through the red supergiant phase (e.g., on a blue loop) to expose first dredgeup abundance ratios, which could quantitatively also explain the observations (dashed line).

More information may be derived from the helium content, which in the case of BA-type supergiants is determined here for a significant number of stars for the first time in a self-consistent analysis. Our results are displayed in Fig. 6. On the MS no helium surface enrichment is observed, as predicted in the models for stars with masses below about $20 M_{\odot}$. After the MS, the picture is blurred by the possible occurrence of a blue loop. Actually the interpretation of the blue supergiant can become really constraining only when we obtain additional hints to the previous evolution of the star. Has the blue supergiant evolved directly from the MS, or has it evolved in that stage after going through a red supergiant stage? At the moment, from the models the situation would be the following: for models below $20 M_{\odot}$, Heenrichments at the level observed in the present supergiants are only compatible with models having undergone a dredge-up in the red supergiant phase. This is true whether rotation is considered or not, or a magnetic field is accounted for or not. The present track for the magnetic $15 M_{\odot}$ model was computed only up to the end of the MS phase and thus did not yet go through the dredge-up phase. Depending on the rotation velocity, the presence of a magnetic field or its absence, models, after the red supergiant phase, will populate diverse parts of the region in the plane $Y_{\mathrm{S}}$ versus $\mathrm{N} / \mathrm{O}$, as illustrated e.g. by the dashed and dotted lines in Fig. 6.

We note, however, that we cannot exclude at present the possibility that the observed helium abundances in the supergiants may be overestimated. A systematic downward shift by a mere $10-15 \%$ (which is within the typical systematic uncertainties in our abundance determinations) would be sufficient, e.g., to bring the observations and the magnetic model in Fig. 6 into agreement. All observed lines arise from two energetically close He I

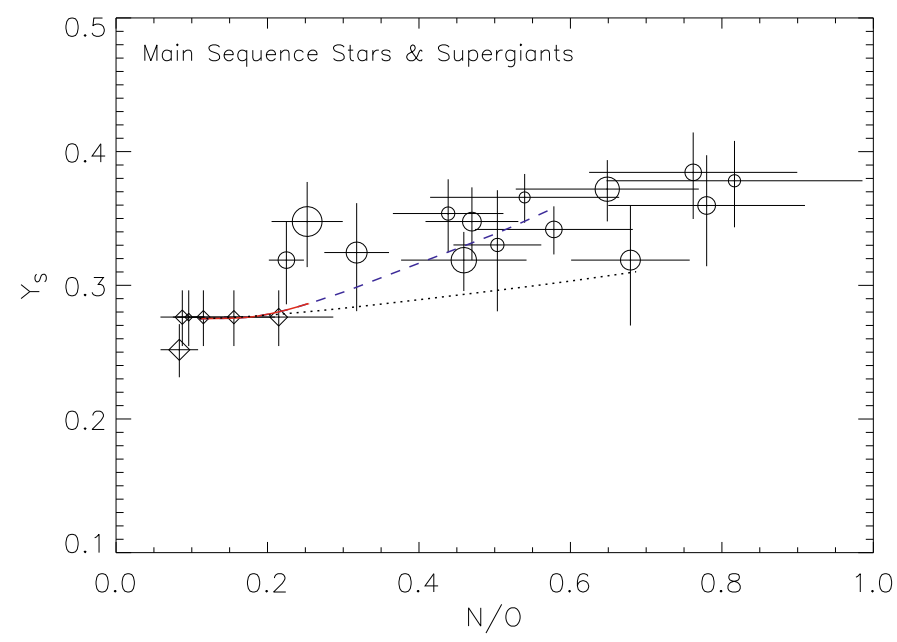

Fig. 6. Surface helium abundance $Y_{\mathrm{S}}$ as a function of N/O ratio (both by mass) for our sample stars. Symbol and line encoding as in Fig. 1.

levels only $\left(2 \mathrm{p}^{1} \mathrm{P}^{\circ},{ }^{3} \mathrm{P}^{\circ}\right)$. Model atom shortcomings (such as insufficient ab-initio collisional data), which may become important only in the weak-line limit at the cool temperature border, could therefore remain unnoticed and could give rise to systematics. It would not be the first time that uncertainties in atomic data complicate the statistic equilibrium and radiative transfer calculations needed to interpret the observed He I lines (e.g. Przybilla 2005; Najarro et al. 2006). Further investigations are required before firm conclusions on the evolutionary state of the supergiants are drawn from the helium abundances, and a fully coherent picture can be deduced.

Finally, an inspection of the total of the $\mathrm{CNO}$ abundances (see Table 1) is instructive. Because the CNO cycles are catalytic, the sum of particles is conserved. Indeed, the sample shows only a small scatter around the mean combined abundance, $\log \sum \mathrm{CNO}+12=8.98 \pm 0.05$. This is further (indirect) evidence that chemical inhomogeneities among the massive star population in the solar neighbourhood are small (Nieva \& Przybilla 2008; PNB08), even at extended distances out to 1$2 \mathrm{kpc}$ from the Sun. Predictions of a high efficiency of hydrodynamic mixing in the interstellar medium and consequently of a locally well-mixed Milky Way (Edmunds 1975; Roy \& Kunth 1995) are thus supported as well.

\section{Conclusions}

Evolution models for massive stars predict very tight relations for the change-of-surface N/O and N/C abundance ratios and for the buildup of helium, as a consequence of mixing with CNO-cycled matter (for given initial chemical composition). Massive stars in the solar neighbourhood, which supposedly share a nearly uniform initial composition (PNB08), are therefore expected to follow the predicted relations, which are governed by nuclear reactions and the dilution effects produced by mixing.

A comparison with NLTE spectral analyses from the literature on CNO abundances in massive stars of $\sim 8-25 M_{\odot}$ leaves room for broad interpretation because of large uncertainties. Observations may even be interpreted as posing a challenge to theory, in particular when the full set of information is not accounted for and conclusions are drawn from one indicator alone, e.g. nitrogen.

On the other hand, our high-precision analyses of a sample of Galactic massive stars from the main sequence to the supergiant 
stage find these tight correlations. Even though further investigations are required to refine the observational constraints on helium abundances before a fully coherent picture can be obtained, the case for strong mixing is clearly supported. It is predicted, e.g., by the models of MM00, by models with magnetic field (MM05), or in cases where the models have gone through the first dredge-up. The separation of the different possibilities may come from studying the evolution of the rotation velocities with time. On the one hand, models with magnetic field predict higher rotational velocities at the end of the MS phase, because the strong internal coupling transmits some of the fast core rotation to the surface. On the other hand, stars on a blue loop should be slower rotators than objects on their first passage from blue to red on average because of the additional angular-momentum loss experienced through strong mass loss during the red supergiant phase. Indeed, some of the slowest rotators show very strong mixing signatures (Table 1).

Finally, we have to point out that some of our results have the potential of challenging the currently available evolution models. The star $\tau$ Sco (HD 149438) stands out in the sample as it shows characteristics that may be explained by a homogeneous evolution, but it requires a highly-efficient spin-down mechanism. One may speculate on magnetic breaking due to angularmomentum losses by a magnetically confined line-driven stellar wind or magnetic coupling to the accretion disc during the starforming process in the case of a fossil field. Even though the topic is not understood theoretically in a comprehensive way, spin-down times of the order of 1 Myr (Ud-Doula et al. 2009) or even less (Mikulášek et al. 2008) are reported for some magnetic massive stars, possibly leading to the required slow rotation already on the zero-age MS in this case as well. Then, if the present helium abundances are confirmed and the supergiants are shown to have evolved directly from the MS, a different kind of mixing may also be required.

We conclude that the tight observational constraints that are required for a thorough testing of the stellar evolution models are within reach. Applications of the improved modelling and analysis techniques to high-quality observations of larger star samples in the Milky Way and other galaxies may finally provide the empirical basis to benchmark the models. It may thus become feasible to disentangle the effects of metallicity, rotation, magnetic fields, and binarity on massive star evolution.

Acknowledgements. N.P. and M.F.N. would like to thank the staff at Geneva Observatory for their hospitality. M.F. acknowledges funding by the Deutsche Forschungsgemeinschaft, DFG under project number PR 685/3-1. Travel to the Calar Alto Observatory/Spain was supported by $D F G$ under grant PR 685/1-1.

\section{References}

Anders, E., \& Grevesse, N. 1989, Geochim. Cosmochim. Acta, 53, 197 Asplund, M., Grevesse, N., Sauval, A. J., \& Scott, P. 2009, ARA\&A, 47, 481 Chiosi, C., \& Maeder, A. 1986, ARA\&A, 24, 329

Crowther, P. A. 2007, ARA\&A, 45, 177

Crowther, P. A., Lennon, D. J., \& Walborn, N. R. 2006, A\&A, 446, 279 Cunha, K., \& Lambert, D. L. 1994, ApJ, 426, 170

Daflon, S., Cunha, K., \& Becker, S. R. 1999, ApJ, 522, 950

Daflon, S., Cunha, K., Becker, S. R., \& Smith, V. V. 2001a, ApJ, 552, 309

Daflon, S., Cunha, K., Butler, K., \& Smith, V. V. 2001b, ApJ, 563, 325

Donati, J.-F., Howarth, I. D., Jardine, M. M., et al. 2006, MNRAS, 370, 629

Edmunds, M. G. 1975, Ap\&SS, 32, 483

Ekström, S., Meynet, G., Maeder, A., \& Barblan, F. 2008, A\&A, 478, 467 (E08)

Firnstein, M. 2006, Diploma Thesis, Univ. Erlangen-Nuremberg

Gies, D. R., \& Lambert, D. L. 1992, ApJ, 387, 673

Grevesse, N., \& Noels, A. 1993, in Origin and Evolution of the Elements, ed.

S. Kubono, \& T. Kajino, 15

Grevesse, N., \& Sauval, A. J. 1998, Space Sci. Rev., 85, 161

Heger, A., \& Langer, N. 2000, ApJ, 544, 1016

Heger, A., Woosley, S. E., \& Spruit, H. C. 2005, ApJ, 626, 350

Herrero, A., Kudritzki, R. P., Vilchez, J. M., et al. 1992, A\&A, 261, 209

Hubrig, S., Briquet, M., De Cat, P., et al. 2009, Astron. Nachr., 330, 317

Hunter, I., Brott, I., Langer, N., et al. 2009, A\&A, 496, 841

Kilian, J. 1992, A\&A, 262, 171

Lyubimkov, L. S. 1996, Ap\&SS, 243, 329

Maeder, A. 1983, A\&A, 120, 113

Maeder, A. 2009, Physics, Formation and Evolution of Rotating Stars (Berlin: Springer Verlag)

Maeder, A., \& Meynet, G. 2000, ARA\&A, 38, 143

Maeder, A., \& Meynet, G. 2005, A\&A, 440, 1041 (MM05)

Maeder, A., Meynet, G., Ekström, S., \& Georgy, C. 2009, CoAst, 158, 72

McErlean, N. D., Lennon, D. J., \& Dufton, P. L. 1999, A\&A, 349, 553

Meynet, G., \& Maeder, A. 2000, A\&A, 361, 101 (MM00)

Meynet, G., \& Maeder, A. 2003, A\&A, 404, 975 (MM03)

Mikulášek, Z., Krtička, J., Henry, G. W., et al. 2008, A\&A, 485, 585

Morel, T., Hubrig, S., \& Briquet, M. 2008, A\&A, 481, 453

Najarro, F., Hillier, D. J., Puls, J., Lanz, T., \& Martins, F. 2006, A\&A, 456, 659

Nieva, M. F., \& Przybilla, N. 2006, ApJ, 639, L39

Nieva, M. F., \& Przybilla, N. 2007, A\&A, 467, 295 (NP07)

Nieva, M. F., \& Przybilla, N. 2008, A\&A, 481, 199

Nieva, M. F., \& Przybilla, N. 2010, in ASP Conf. Ser., 425, 146

Przybilla, N. 2005, A\&A, 443, 293

Przybilla, N., Butler, K., Becker, S. R., \& Kudritzki R. P. 2006, A\&A, 445, 1099

Przybilla, N., Nieva, M. F., \& Butler K. 2008, ApJ, 688, L103 (PNB08)

Roy, J.-R., \& Kunth, D. 1995, A\&A, 294, 432

Schiller, F., \& Przybilla, N. 2008, A\&A, 479, 849

Schönberner, D., Herrero, A., Becker, S. R., et al. 1988, A\&A, 197, 209

Searle, S. C., Prinja, R. K., Massa, D., \& Ryans, R. 2008, A\&A, 481, 777

Spruit, H. C. 2002, A\&A, 381, 923

Takeda, Y. 2000, PASJ, 52, 113

Ud-Doula, A., Owocki, S. P., \& Townsend, R. H. D. 2009, MNRAS, 392, 1022

Venn, K. A. 1995, ApJ, 449, 839

Venn, K. A., \& Przybilla, N. 2003, in ASP Conf. Ser., 304, 20

Walborn, N. R. 1976, ApJ, 205, 419 